\title{
Postsurgical Cystoid Macular Edema following Posterior Chamber Toric Phakic Intraocular Lens Implantation Surgery: A Case Report
}

\author{
Julide Canan Umurhan Akkan Kemal Tuncer Ahmet Elbay \\ Department of Ophthalmology, Faculty of Medicine, Bezmialem Vakif University, \\ Istanbul, Turkey
}

\section{Key Words}

Postsurgical cystoid macular edema - Posterior chamber intraocular lens - Toric implantable collamer lens $\cdot$ Intraocular lens complication

\begin{abstract}
Purpose: To describe a case of cystoid macular edema (CME) developing after posterior chamber toric phakic intraocular lens (PIOL) implantation. Methods: Case report. Results: A 33-year-old male underwent implantation of toric implantable collamer lenses (ICL), a new generation of PIOLs, for both eyes. Preoperative best spectacle-corrected distance visual acuity (BCVA) was 20/25 in the right eye and 20/32 in the left eye, with a manifest refraction of $-9.25-4.0 \times 4^{\circ}$ and $-9.75-4.25 \times 171^{\circ}$, respectively. On day 1 postoperatively, the left eye had an uncorrected distance visual acuity (UDVA) of 20/60 with a refraction of $+2.0-3.5 \times$ $11^{\circ}$. Despite the rotation of the PIOL, the cylindrical refractive component persisted in the left eye with a refraction of $+2.0-3.5 \times 11^{\circ}$. Two weeks after the initial surgery, he presented with a decrease in his visual acuity in the left eye. The UDVA and BCVA were both 20/100 in the left eye with a refraction of $+2.0-4.25 \times 3^{\circ}$. Dilated fundus examination and macular optical coherence tomography revealed a CME in the left eye. Following topical nepafenac therapy and explantation of the ICL, we observed a complete resolution of the CME at 3 months with an improvement in BCVA to 20/32 in the left eye. Conclusions: To our knowledge, this is the first reported case of postsurgical CME following toric ICL implantation. In cases of phakic eyes with an intact posterior capsule, postsurgical CME can develop, thus highlighting the purpose of this report.

(C) 2015 S. Karger AG, Basel
\end{abstract}

KARGER 125/s $\quad \begin{aligned} & \text { Julide Canan Umurhan Akkan, MD } \\ & \text { Faculty of Medicine, Bezmialem Vakif University } \\ & \text { Adnan Menderes Bulvarı (Vatan Cad.) } \\ & \text { TR-34093 Fatih/İstanbul (Turkey) } \\ & \text { E-Mail jumurhan@hotmail.com }\end{aligned}$


Umurhan Akkan et al.: Postsurgical Cystoid Macular Edema following Posterior Chamber Toric Phakic Intraocular Lens Implantation Surgery: A Case Report

\section{Introduction}

Phakic intraocular lenses (PIOLs) have been shown to be an effective surgical option for the treatment of ametropia of various refractive ranges, offering an optical quality claimed to be superior to that of corneal refractive surgeries [1-3]. The Visian implantable collamer lens (ICL; STAAR Surgical, Nidau, Switzerland) is the only posterior PIOL that is currently approved by the United States Food and Drug Administration (FDA) for the treatment of moderate to severe myopia, while the toric version was under review with the FDA. The efficacy and safety of the ICL has been demonstrated in several studies $[2,4,5]$. Posterior segment complications including rhegmatogenous retinal detachment after ICL surgery have been reported $[2,6]$. To our knowledge, there have been no reports of cystoid macular edema (CME) as a complication of ICL implantation. We present a patient with postsurgical CME demonstrated by optical coherence tomography (OCT) arising after toric ICL implantation.

\section{Case Report}

A 33-year-old male diagnosed with myopia presented for the evaluation of refractive surgery. His medical and ophthalmological histories were unremarkable. Best spectaclecorrected distance visual acuity (BCVA) was 20/25 in the right eye and 20/32 in the left eye, with a manifest refraction of $-9.25-4.0 \times 4^{\circ}$ and $-9.75-4.25 \times 171^{\circ}$ in the right and left eye, respectively. Ocular examination including slit-lamp biomicroscopy and indirect ophthalmoscopy was normal in both eyes. The intraocular pressure was $13 \mathrm{~mm} \mathrm{Hg}$ in the right eye and $12 \mathrm{~mm} \mathrm{Hg}$ in the left eye. Corneal topography was performed using the rotating Scheimpflug camera (Pentacam; Oculus Surgical, Inc., Port St. Lucie, Fla., USA). Keratometry values for the steep and flat axis were $47.25 \mathrm{dpt}$ at $94^{\circ}$ and $43.7 \mathrm{dpt}$ at $7^{\circ}$, respectively, in the right eye, and $46.8 \mathrm{dpt}$ at $84^{\circ}$ and $43.6 \mathrm{dpt}$ at $174^{\circ}$, respectively, in the left eye. The anterior chamber depth of both eyes was 3.27 and $3.25 \mathrm{~mm}$, and central corneal thickness was 492 and $490 \mu \mathrm{m}$, respectively. Biometry was performed with the IOL Master (Carl Zeiss Meditec, Jena, Germany). Axial length measured 26.84 and $26.89 \mathrm{~mm}$ for the right and left eyes, respectively. White-to-white measurements with callipers were $12 \mathrm{~mm}$ in both eyes. Compared with the previous data, his refractive error and keratometry values had been stable during the previous 12 months. Toric PIOLs (Visian ICL with Centraflow; STAAR Surgical) were chosen for implantation, aiming for postoperative plano-refraction in both eyes (VTICM013.2 SN:D170993 for the right eye, VTICM013.2 SN:D170660 for the left eye). The patient underwent toric ICL implantation under general anesthesia in the right eye without complications (fig. 1). On day 1 postoperatively, the uncorrected distance visual acuity (UDVA) was 20/25. One week later, the patient proceeded to surgery for the left eye. Left eye toric ICL implantation software suggested rotating the lens $10^{\circ}$ counterclockwise after horizontal implantation (lens label data $-14.0 /+4.5 / 073$ ). Tobramycin and dexamethasone $0.1 \%$ eye drops 4 times a day for 10 days were used for both eyes postoperatively. On the first postoperative day, the left eye had a UDVA of $20 / 60$ with a refraction of $+2.0-3.5 \times 11^{\circ}$. We observed a rotation of toric ICL of approximately $10^{\circ}$ from its original position in the left eye and decided to plan the repositioning due to the fact that the patient was unsatisfactory with his surgical outcome. The following day after rotating, we did not found a noticeable improvement in visual acuity with a refraction of $+2.0-4.25 \times 3^{\circ}$. The position of the ICL was excellent with an adequate vault. Repeat postoperative keratometry was consistent with preoperative readings. Since other possibilities were ruled out, we suspected wrong labeling or producing of the ICL by the manufacturer. Before the discussion of the findings with the 
Umurhan Akkan et al.: Postsurgical Cystoid Macular Edema following Posterior

Chamber Toric Phakic Intraocular Lens Implantation Surgery: A Case Report

patient, he contacted the clinic due to a decrease in visual acuity of the left eye 1 week later. The UDVA and BCVA were both 20/100 in the left eye with a refraction of $+2.0-4.25 \times 3^{\circ}$. Slit-lamp examination of the anterior segment was unremarkable, while dilated fundus examination revealed a CME in the left eye. Macular OCT on the left eye showed hyporeflective intraretinal cystoid cavities with subretinal fluid composed of retinal elevation. Foveal thickness was $189 \mu \mathrm{m}$ in the right eye and $529 \mu \mathrm{m}$ in the left eye. Nepafenac ophthalmic solution was prescribed 3 times a day for 4 weeks. The patient was advised of ICL explantation, to which he agreed. During the first week of the nepafenac therapy, an explantation surgery of the toric ICL was performed in the left eye. Three weeks later, foveal thickness was $308 \mu \mathrm{m}$ in the left eye. OCT showed a complete resolution of the CME at 3 months following explantation of the ICL (fig. 2) with an improvement in BCVA to 20/32 in the left eye. The patient underwent a new toric ICL (VTICM013.2, SN: T103450, label data: $13.5 /+3.5 / 087)$ implantation in the left eye approximately 6 months after the initial implantation. UDVA was $20 / 30$ in the left eye with a refraction of $+0.25-0.50 \times 60^{\circ}$, where it remained stable for the next 6 months.

\section{Discussion}

Postsurgical CME (resulting in Irvine-Gass syndrome) is the most common cause of vision loss after phacoemulsification and one of the main causes of reduced vision in other ocular surgeries, including vitreoretinal procedures, penetrating keratoplasty, and Nd-YAG laser capsulotomy [7]. Although its etiology is not completely understood, direct macular traction and postoperative inflammatory processes have been proposed as causative agents. Following cataract surgery, inflammatory mediators disrupt the blood-retinal barrier, increasing the permeability of the perifoveal capillaries and resulting in fluid accumulation in the perifoveal retina [8]. There are also reports on CME following anterior chamber and irissupported PIOLs [9]. To our knowledge, this is the first report of postsurgical CME following ICL, a new generation posterior chamber PIOL, implantation surgery.

Posterior chamber PIOLs are vaulted between the posterior pigmented layers of the iris and the anterior crystalline lens with the anterior zonules. The mechanisms for developing CME may include constant friction between the posterior iris surface and the phakic lens or between the haptic and the ciliary sulcus. However, we observed the CME during the early postoperative period. A PIOL insertion may also induce traction on the anterior and/or posterior vitreous base, causing CME. A consecutive surgical intervention of repositioning following the initial surgery probably increased the risk of CME development.

Toric ICL rotation is considered to be around $2.06^{\circ}( \pm 4.25)$ depending on the case series $[5,10]$. Navas et al. [11] presented a case of toric ICL spontaneous rotation of $35^{\circ}$ from its original surgical position 3 months after surgery. The angle of the rotation in our case was smaller (approx. $10^{\circ}$ ) than in their case. We assumed that this amount of rotation affected the manifest refraction in our case, causing to plan for repositioning. The cylindrical refractive component with an increase of $0.75 \mathrm{dpt}$, however, still persisted after the repositioning. It has been reported that an incorrectly labeled intraocular lens must be considered a potential cause of the unintended refractive outcome in cases in which no other cause for a significant refractive surprise has been identified [12]. In the present case, we suspected that the toric ICL had been incorrectly labeled or produced (no cylindrical power). Unfortunately, there has been no definitive proof of the casual link between the postoperative refractive outcome and the power of the implanted toric ICL. We were not able to obtain a confirmation by the manufacturer. 
Umurhan Akkan et al.: Postsurgical Cystoid Macular Edema following Posterior

Chamber Toric Phakic Intraocular Lens Implantation Surgery: A Case Report

In routine practice, we did not prefer to administer a topical nonsteroidal antiinflammatory drug (NSAID) after PIOL procedures. Additionally, there were no systemic and/or ophthalmic risk factors such as diabetes, retinal vascular diseases, uveitis, etc. for CME in the present case. Currently, medical prophylaxis is not approved for normal eyes without previous problems [7]. However, it can also be discussed if the highly myopic eyes that underwent PIOL implantation surgery are 'normal' eyes. In spite of the fact that the accompanying visual loss in postoperative CME is usually self-limiting, one should be aware of the development of CME following ICL implantation. One case report cannot suggest using topical NSAID therapy for preventing CME after PIOL implantation in eyes with myopia but should alert the surgeons to closely follow the patients for early detection of CME, especially in complicated cases.

\section{Statement of Ethics}

The patient's informed consent has been obtained.

\section{Disclosure Statement}

The authors have no conflicts of interest to report.

\section{References}

1 Sanders DR, Doney K, Poco M: United States Food and Drug Administration clinical trial of the Implantable Collamer Lens (ICL) for moderate to high myopia: three-year follow-up. Ophthalmology 2004;111:16831692.

2 Igarashi A, Shimizu K, Kamiya K: Eight year follow-up of posterior chamber phakic lens implantation for moderate to high myopia. Am J Ophthalmol 2014;157:532-539.

-3 Kamiya K, Igarashi A, Shimizu K, et al: Visual performance after posterior chamber phakic intraocular lens implantation and wavefront - guided laser keratomileusis for low to moderate myopia. Am J Ophthalmol 2012;153:1178-1186.

4 Alfonso JF, Lisa C, Fernández-Vega L, et al: Posterior chamber collagen copolymer phakic intraocular lenses to correct myopia: five year follow-up. J Cataract Refract Surg 2015;41:800-805.

5 Sanders DR, Schneider D, Martin R, et al: Toric implantable collamer lens for moderate to high myopic astigmatism. Ophthalmology 2007;114:54-61.

6 Martinez-Castillo V, Boixadera A, Verdugo A, et al: Rhegmatogenous retinal detachment in phakic eyes after posterior chamber phakic intraocular lens implantation for severe myopia. Ophthalmology 2005;112:580585.

7 Loewenstein A, Dinah Z: Postsurgical cystoid macular edema; in Coscas G (ed): Macular Edema. Dev Ophthalmol. Basel, Karger, 2010, vol 47, pp 148-159.

8 Miyake K, Ibaraki N: Prostaglandins and cystoid macular edema. Surv Ophthalmol 2002;47(suppl 1):203218.

-9 Lovisolo CF, Reinstein DZ: Phakic intraocular lenses. Surv Ophthalmol 2005;50:549-587.

10 Hashem AN, El Danasoury AM, Anwar HM: Axis alignment and rotational stability after implantation of the toric implantable collamer lens for myopic astigmatism. J Refract Surg 2009;25:939-943.

-11 Navas A, Munos-Ocampo M, Graue-Hernandez EO, et al: Spontaneous rotation of a toric implantable collamer lens. Case Rep Ophthalmol 2010;1:99-104.

$\checkmark 12$ Solebo LA, Eades-Wolker RJ, Dabbaqh A: Intraocular lens exchange for pseudophakic refractive surprise due to incorrectly labeled intraocular lens. J Cataract Refract Surg 2012;38:2197-2198. 


\section{Case Reports in \\ Ophthalmology}

\begin{tabular}{l|l}
\hline \multicolumn{2}{l}{ Case Rep Ophthalmol 2015;6:223-227 } \\
\hline DOI: 10.1159/000437013 & $\begin{array}{l}\text { @ 2015 S. Karger AG, Basel } \\
\text { www.karger.com/cop }\end{array}$ \\
\hline
\end{tabular}

Umurhan Akkan et al.: Postsurgical Cystoid Macular Edema following Posterior

Chamber Toric Phakic Intraocular Lens Implantation Surgery: A Case Report

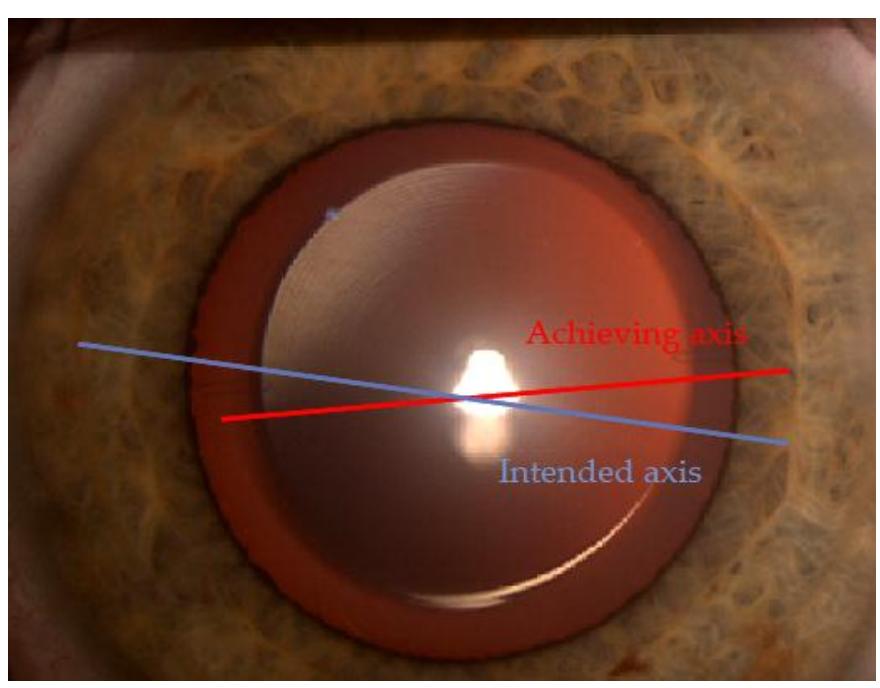

Fig. 1. Slit-lamp image of the ICL in the left eye on day 1 postoperatively.
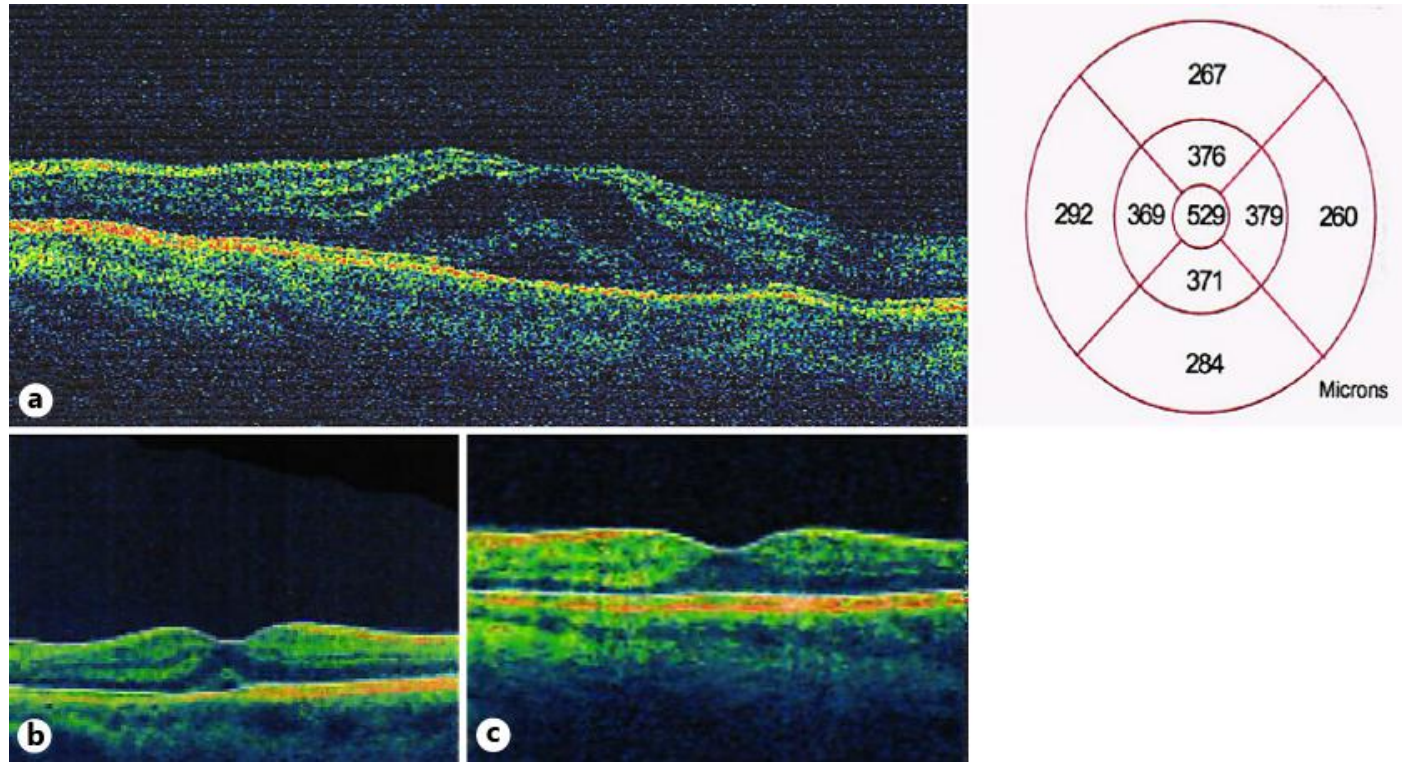

Fig. 2. a Postoperative OCT scan of the CME following ICL implantation. $\mathbf{b}$ OCT scan of the macula 3 weeks later following topical nepafenac therapy. Central retinal thickness was $308 \mu \mathrm{m}$. c OCT scan of the resolved CME at the last visit. 